\title{
Scan-to-Pattern Clothing Systems: A Systematic Approach
}

\author{
Bruce BRADTMILLER* \\ Anthrotech Inc., Yellow Springs $\mathrm{OH}$, USA \\ http://dx.doi.org/10.15221/13.379
}

Sizing systems for commercial off-the-rack clothing have historically been based on a mix of tradition, trade secrets and anthropometric data. More recently, large quantities of information from 3D body scanners have become available, creating a new source of data from which off-the-rack clothing can be sized and designed. [We note that some scanning systems are used for made-to-measure or custom garments, and those processes are not the subject of this paper. This paper will also not deal with the direct use of the 3-D image to create patterns without the extraction of dimensions.] With these new, large, datasets available it is worthwhile to review the whole process of how to go from a scanner-produced anthropometric dataset to finished patterns.

\section{Validating the scanner and its extraction software}

The scanner itself should be validated for this particular application. Research has shown that scanners vary in the quality of the scan image, and the amount of missing data (as well as the software tools to estimate the missing data) (Bradtmiller and Gross, 1999, 2003; Chang, 2007; Daanen, 1997a; 1997b; 1997c; Kouchi and Mochimaru, 2006a, 2006b, 2008; 2011; Paquette et al., 2000; van Stralen et al., 2003; Suikerbuik et al., 2004). Systems also vary considerably in the algorithms they use to extract the anthropometric dimensions. Leaving aside whether the extracted dimensions are "right" or "wrong", it is important to know whether they are providing the dimensions that the user expects. Ideally, this validation should be done by an independent agency, but at minimum should not be done by the system manufacturer.

ISO 20685 "3-D scanning methodologies for internationally compatible anthropometric databases" (ISO, 2010) outlines a systematic method for validating the extracted dimensions from 3-D scanners. Since humans almost constantly change size and shape, it is not possible to measure the body with the same accuracy that could be achieved in, say, a piece of machine tooling. In that particular standard, the accuracy of the dimensions extracted from the scanner is compared with the level of repeatability achieved by skilled human anthropometrists. As some dimensions are more difficult than others to measure-for both the human and the software application-the required level of accuracy varies with the type of dimension. For segment lengths, for example, the standard is set at $5 \mathrm{~mm}$, while the larger circumferences like chest and waist, the standard is set at $9 \mathrm{~mm}$. The standard goes on to outline research designs for setting up a demonstration to show that a given scanner and extraction algorithm performs to the necessary level of accuracy. It includes information about the sample size, sample selection and the analytical procedures which ought to be used.

\section{Creating the sample dataset}

When the scanner is validated, the next step is to actually collect the data, by scanning a sample from the population of interest. The population might be a national group, a trading zone, such as the European Union, or it might be a specific market segment, such as young women from 18 to 30 . The various ways to acquire the sample and avoid sampling bias is the topic for another paper. For the present purpose, we will assume that the data have been appropriately collected from an appropriately defined population of interest. The definition of the population will be in terms of one or more demographic characteristics, such as racial/ethnic group, age, occupation, household income, etc.

\section{Data editing and weighting}

An often-overlooked piece of the data stream is the editing stage. In every data collection enterprise, whether it is a very traditional tape and caliper project, or whether it makes use of the latest scanning technology such as that seen at this conference, some unusual values will occur. Some of those unusual values may be due to some unusual characteristic of the person being measured or scanned. But, other unusual values will be the result of errors, which could be made by a human measurer, or could be made by the measurement extraction algorithm when processing the scan data. In either case, it is important to distinguish between unusual values because the subject is unusual, and those which are errors due to the measurement system. Identifying and correcting or removing these erroneous values is called data editing, and there are a number of data editing methods.

\footnotetext{
*Anthrotech, Inc., 503 Xenia Avenue, Yellow Springs, OH 45387; bruce@anthrotech.net
} 
Ones that we have found useful are described in Kikta and Churchill (1977) and Churchill (et al., 1988). They involve a two-step process in which population outliers are first identified, and then values are checked against each other within each individual to look for reasonableness.

The final step in preparation of the working database is to correct for any deficiencies in sample acquisition. In nearly every case in my experience, the actual measured or scanned sample does not exactly match the sampling goal. As long as the scanned sample is reasonably close to the target, it is possible to statistically weight the data set to make it match the demographic characteristics of the target population.

The weights are calculated as the relative frequency of a given cell in the target population, divided by the relative frequency of the same cell in the sample. For example, in a sampling plan with two characteristics such as racial group and age group, it can be expressed as:

$$
\begin{aligned}
& {\left[N_{1,1} /\left(N_{1,1}+N_{1,2}+\ldots+N_{i, j}\right)\right] /\left[n_{1,1} /\left(n_{1,1}+n_{1,2}+\ldots+n_{i, j}\right)\right]} \\
& \text { where } N \text { is the count from the demographic cell in the target, } \\
& n \text { is the count from the age/race cell in the sample, } \\
& i \text { is the subscript for the last age group, and } \\
& j \text { is the subscript for the last racial group. }
\end{aligned}
$$

Sampling cells which are under-represented will have a higher statistical weight. Cells in the sample that are over-represented will have a lower statistical weight. Using the weighted anthropometric values when calculating any final statistics or conducting other analyses will approximate the true anthropometric values in the target population as a whole.

Another approach to solving problem of non-representativeness of samples was pioneered by Parkinson and Reed (2009), who developed a method to create a virtual population with the required demographic characteristics. Using a combination of principal component analysis and linear regression, they create statistical models that draw on the anthropometric relationships in a population with many dimensions, to match the demographic distribution of a population (the target) with some but fewer anthropometric dimensions. Their demonstration creates a virtual database by using two different populations, but it could be equally well applied in the current context, where the population of interest has been sampled, but sampled imperfectly.

\section{Creating the sizing system}

The next step in an anthropometric sizing system is to determine the key dimensions. These are the dimensions according to which an individual will identify which size to purchase. In most cases these are a matter of tradition: men's dress shirts, for example, are sized by neck circumference and sleeve length. Men's trousers are sized on waist circumference and inseam. For totally new garments, or for some types of one-piece garments, such as a protective coverall, it is necessary to examine the advantages and disadvantages of a set of key dimensions. For example, in the context of the sale, will a customer actually take body measurements? Or should we restrict our consideration to measurements that customer is likely to know without measuring, such as the waist? The one-piece coverall may best be sized by a vertical trunk circumference, with a tape passing over the shoulder and through the crotch. But this is a measurement that most people do not know for themselves, so unless the customer is likely to measure it before selecting a garment, then another key dimension should probably be used.

Selecting a dimension is a statistical as well as a practical matter. Statistically, the dimensions of choice are those which are highly correlated with other important garment design dimensions. In the case of a pilot's G-suit that pushes blood towards the head during a plane's rapid changes of direction, either waist or hip is equally effective in its association with the other design dimensions. As a practical matter, more individuals know their waist size than their hip size, so we would select waist circumference as the primary sizing dimension. For length, we would statistically examine the possible dimensions. In the particular example, we found that both leg inseam and leg outseam were better predictors of critical garment dimensions than was stature. Between leg inseam and outseam, inseam has the advantage that many men know (from slacks purchases) what their inseam is. From a pattern-making perspective, however, that dimension would pose difficult challenges since the Gsuit is essentially crotchless, and it would be difficult to associate human dimensions with garment dimensions without the seam at the crotch. We would therefore chose leg outseam (waist height at the navel) in that case as the length key dimension. 
We next examine how much the key dimensions change per size. Sometimes this is a matter of tradition, as in the example of men's dress shirts. The neck is typically graded by $1 / 2$ inch, so grading by $3 / 4$ inch would have a difficult time in the marketplace, even if a perfectly adequate it could be achieved. In other cases, especially on a new garment, it is not clear what the grade should be. In those cases, it is helpful to have a prototype of a central size, and to conduct a small fit test to determine the full range of body sizes and shapes that fit into a single size. This process, sometimes called "fit-mapping" (Robinette, 2000), identifies through empirical data collection the upper and lower limits of body size for each key dimension in the garment size. It should be noted that the grade for each of the key dimensions is not necessarily the same. In the dress shirt example, while the neck is graded at $1 / 2$ inch, the sleeves are usually graded at 1 or even 2 inches. If there is a third dimension, such as the difference between the chest circumference and the waist circumference, the "drop", that third dimension may simply sort customers into 2 or 3 broad categories, so it may have a grade of several inches.

When the accommodation range for a single size is known, then it is relatively straightforward to place the single size on the anthropometric distribution of the sample, and then place additional sizes until the accommodation goal for the population is met. This process determines both the grade of the key dimensions, and the total number of sizes needed to accommodate the population. Sometimes, business factors such as shelf space, rather than quality of fit, determine the number of sizes that are eventually produced.

With the sizing system established, the final step in anthropometric sizing is to determine which values of the design dimensions are to be used in the actual garment design. If there is a sufficient sample of people in each of the sizes, we would begin by calculating, for each of the sizes, the basic anthropometric summary statistics for subset of the sample in that size. The potential design dimensions for the $G$ suit are seen in Table 1, for the size Small-Short. A similar table would be created for each of the sizes.

Table 1. Sizing Dimensions: Small-Short (values in inches)

\begin{tabular}{|l|r|r|r|r|r|}
\hline DIMENSION & MINIMUM & $\begin{array}{c}\text { 5TH } \\
\text { \%ILE }\end{array}$ & MEAN & \multicolumn{1}{c|}{$\begin{array}{c}\text { 95TH } \\
\text { \%ILE }\end{array}$} & MAXIMUM \\
\hline Ankle Circumference & 7.2 & 7.8 & 8.4 & 9.1 & 9.8 \\
\hline Ankle-Knee Length & 14.7 & 15.5 & 16.4 & 17.5 & 18.4 \\
\hline Buttock Circumference & 31.8 & 34.3 & 36.7 & 39.0 & 42.0 \\
\hline Buttock Height & 30.4 & 31.9 & 33.5 & 35.1 & 36.3 \\
\hline Calf Circumference & 10.9 & 13.0 & 14.2 & 15.5 & 16.2 \\
\hline Calf Height & 11.4 & 12.3 & 13.3 & 14.3 & 15.1 \\
\hline Crotch Height & 28.5 & 30.0 & 31.7 & 33.1 & 34.2 \\
\hline Gluteal Furrow Height & 27.8 & 29.0 & 30.6 & 32.2 & 32.9 \\
\hline Knee-Buttock Length & 12.8 & 13.5 & 14.5 & 15.5 & 16.6 \\
\hline Knee Circumference & 12.3 & 13.5 & 14.5 & 15.6 & 16.3 \\
\hline Knee Height & 17.2 & 18.0 & 19.0 & 20.0 & 20.7 \\
\hline Lateral Malleolus Height & 2.0 & 2.2 & 2.6 & 2.9 & 3.1 \\
\hline Lower Thigh Circ & 12.2 & 13.3 & 14.6 & 16.0 & 17.3 \\
\hline Tenth Rib Height & 39.5 & 40.6 & 42.3 & 43.8 & 44.6 \\
\hline Thigh Circ & 17.8 & 19.8 & 22.0 & 24.1 & 26.1 \\
\hline Waist (NI) Circ & 26.5 & 28.0 & 30.6 & 32.8 & 34.1 \\
\hline Wasit (O) Circ* & 27.5 & 28.3 & 31.0 & 33.2 & 33.5 \\
\hline Waist (NI) Height & 39.1 & 40.6 & 42.5 & 44.0 & 44.7 \\
\hline Waist (O) Height* & 37.6 & 38.2 & 40.1 & 41.3 & 41.5 \\
\hline Waist (NI) Ht-Waist (O) Ht & 0.9 & 1.5 & 2.4 & 3.4 & 4.0 \\
\hline
\end{tabular}

${ }^{*}$ Key dimensions 
If the sample data set is not large enough to have adequate representation in each of the sizes for single-size summary statistics, then a linear regression method would be used to essentially take into account all the change in a given dimension from the smallest to the largest size, and proportion it equally over all the sizes. This is more similar to a standard grading approach.

Choosing the actual design dimensions - that is, the anthropometric dimensions of the fit model for that size-requires some understanding of the garment itself, some understanding of the fabric characteristics, and some understanding of the underlying body size variability. There is no formulaic approach that will be suitable in every case. In the G-suit example, for the circumferences (waist, hip, thigh, calf, etc.) we elected to design close to the maximum values seen in a size. We made this choice because a smaller person can be accommodated in a too-large garment, while the reverse is not true in a non-stretch fabric. Further, in selecting the maximum as opposed to the commonly used 95th percentile, we recognized that for this garment, a custom fit garment is not a realistic possibility. Thus, the sizing system simply must accommodate everyone in the population.

For the lengths, we designed to the middle of the range (mean value). Since the garment is essentially anchored at the waist, any excess length would not be spread over all the legs, but would be forced downward. This is especially critical in the location of the crotch cutout and the knee cutout. If these are misplaced (a problem in earlier versions of the garment) extreme discomfort will result. It is less of a problem if these cutouts are too high. In other garments, different decisions would be made.

When the design values for each of the sizes are chosen, typically a summary table is created, showing either the specific design values for each size, or a central size with grading increments for each body dimension. And, it should be noted that all the work to this point has been on body dimensions and not on garment dimensions.

\section{Creating the patterns}

The next step in the anthropometric sizing and patterning is to create the actual patterns. At this point, the designers and pattern-makers can work in their usual way. If there was a prototype used for fitmapping, then that becomes the central size, and the other sizes are created from there. If not, the designer and pattern-maker would create a prototype based on a living fit model whose dimensions match as closely as possible the body dimensions for the central size. After the prototype is optimized through the designer's usual process, then the pattern can be graded using the tables created in the previous step.

\section{Validating the sizing and grading}

A final piece is to validate sizing and grading by conducting a fit test. For a totally new garment, there would ideally be a test article in each size. For garments that are made routinely, typically only a few sizes-perhaps the central size, and sizes near the upper and lower end of the range-would be sufficient. Each size of the test articles should be tested by several participants (5 is a good minimum number) to verify that the sizing and grading functioned as intended.

\section{Conclusion}

A process has been outlined here to start with scanner-generated anthropometric data, and end with successful, tested patterns from a systematically developed sizing system. This systematic approach will allow a traceable path from scan data to verified patterns. Because it is systematic, it is amenable to changes in the process for specific garments, specific design populations or other unusual circumstances. It also allows designers update the sizing system as the population changes over time. It has been used on a number of garments, from dress clothing to functional and protective clothing, and has been successful each time.

\section{References}

Bradtmiller, Bruce and Mary E. Gross (1999) "3D Whole Body Scans: Measurement Extraction Software Validation", SAE Technical Paper Series, Digital Human Modeling for Design and Engineering, International Conference and Exposition, The Hague, The Netherlands. 
Bradtmiller, Bruce and Mary E. Gross (2003) "Anthropometric Dimensions Extracted from Surface Scans and Measured Directly: Issues for Standardization", Proceedings of the XVth Triennial Congress of the International Ergonomics Association and 7th Joint Conference of Ergonomics Society of Korea and Japan, Seoul, Republic of Korea.

Chang, Chien-Chi, Zhizhong Li, Xiuwen Cai and Patrick Dempsey (2007) Error Control and Calibration in Three-Dimensional Anthropometric Measurement of the Hand By Laser Scanning With Glass Support, Measurement 40:21-27.

Churchill, Thomas D., Bruce Bradtmiller and Claire C. Gordon (1988) Computer software used in U.S. Army anthropometric survey 1987-1988. TR NATICK/TR-88/045. U.S. Army Natick Research, Development and Engineering Center, Natick, MA 01760-5000.

Daanen, H.A.M., M.A. Brunsman and K.M. Robinette (1997) "Reducing movement artifacts in whole body scanning", IEEE Computer Graphics and Applications, Volume 0-8186-7943-3/97: 262-265.

Daanen, Hein, Matt Brunsman and Stacie Taylor (1997) "Absolute accuracy of the Cyberware WB4 whole body scanner", contract report to Crew Systems Directorate, Armstrong Laboratory, WrightPatterson Air Force Base, OH, Contract Number F41624-93-C-6001.

Daanen, H.A.M. and A.J. Krul (1997) "Reliability of circumference estimation based on depth and width of the hip. TNO-report TM-97-A088. TNO Human Factors Research Institute, Soesterberg, The Netherlands.

ISO (2010) ISO 20685 3-D scanning methodologies for internationally compatible anthropometric databases. International Standards Organisation Geneva.

Kikta, Paul and Thomas Churchill (1978) Editing procedure for anthropometric survey data. AMRLTR-78-38. Aerospace Medical Research Laboratory, Aerospace Medical Division, Air Force Systems Command, Wright-Patterson Air Force Base, OH 45433.

Kouchi, Makiko and Masaaki Mochimaru (2011) Errors in landmarking and the evaluation of the accuracy of traditional and 3D anthropometry. Applied Ergonomics 42(3): 518-527.

Kouchi, Makiko and Masaaki Mochimaru (2006) "Why scan-derived body measurements differ from measurement by the traditional methods?", in Proceedings of the International Ergonomics Association 2006 Congress, E.A.P. Koningsveld, R.N. Pikaar and P.J.M. Settels, eds.. Elsevier.

Kouchi, Makiko and Masaaki Mochimaru (2006) "Quality Control of Anthropometric Data", in Proceedings of the International Ergonomics Association 2006 Congress, E.A.P. Koningsveld, R.N. Pikaar and P.J.M. Settels, eds.. Elsevier.

Kouchi, Makiko and Masaaki Mochimaru (2008) "Evaluation of accuracy in traditional and 3D anthropometry", paper presented at Digital Human Modeling for Design and Engineering Conference, Pittsburgh, PA.

Paquette, Steven, David J. Brantley, Brian D. Corner, Peng Li and Thomas Oliver (2000) "Automated extraction of anthropometric data from 3D images", XIVth Triennial Congress of the International Ergonomics Association and the 44th Annual Meeting of the Human Factors and Ergonomics Society, San Diego, CA.

Parkinson, M.B. and Reed, M.P. (2009). Creating virtual user populations by analysis of anthropometric data. International Journal of Industrial Ergonomics, 40:106-111.

Robinette, Kathleen M. (2000) "3-D Fit Mapping", XIVth Triennial Congress of the International Ergonomics Association and the 44th Annual Meeting of the Human Factors and Ergonomics Society, San Diego, CA.

Suikerbuik, Rob, Hans Tangelder, Hein Daanen and Aurnout Oudenhuijzen (2004) "Automatic feature detection in 3D human body scans", Digital Human Modeling and Engineering Symposium, Rochester, MI.

Van Stralen, M., H.A.M. Daanen and J.W.H. Tangelder (2003) "A tool box to identify holes in 3D human body scans", Proceedings of the XVth Triennial Congress of the International Ergonomics Association and 7th Joint Conference of Ergonomics Society of Korea and Japan, Seoul, Republic of Korea. 